\title{
Existence Results for Nonsmooth Vector Quasi-Variational-Like Inequalities
}

\author{
Mohammed Alshahrani, ${ }^{1}$ Qamrul Hasan Ansari, ${ }^{1,2}$ and Suliman Al-Homidan ${ }^{1}$ \\ ${ }^{1}$ Department of Mathematics and Statistics, King Fahd University of Petroleum \& Minerals, Dhahran 31261, Saudi Arabia \\ ${ }^{2}$ Department of Mathematics, Aligarh Muslim University, Aligarh 202 002, India
}

Correspondence should be addressed to Mohammed Alshahrani; mshahrani@kfupm.edu.sa

Received 27 June 2013; Accepted 1 August 2013

Academic Editor: Jen-Chih Yao

Copyright (c) 2013 Mohammed Alshahrani et al. This is an open access article distributed under the Creative Commons Attribution License, which permits unrestricted use, distribution, and reproduction in any medium, provided the original work is properly cited.

\begin{abstract}
We introduce nonsmooth vector quasi-variational-like inequalities (NVQVLI) by means of a bifunction. We establish some existence results for solutions of these inequalities by using Fan-KKM theorem and a maximal element theorem. By using the technique and methodology adopted in Al-Homidan et al. (2012), one can easily derive the relations among these inequalities and a vector quasi-optimization problem. Hence, the existence results for a solution of a vector quasi-optimization problem can be derived by using our results. The results of this paper extend several known results in the literature.
\end{abstract}

\section{Introduction}

The theory of quasi-variational inequalities (QVI) was started with a pioneerwork of A. Bensoussan and J. L. Lions in 1973, perhaps motivated by the stochastic control and impulse control problems. It was the paper of Bensoussan et al. [1] in which the term quasi-variational inequality was introduced. The quasi-variational inequality is an extension of a variational inequality [2] in which the underlying set depends on the solution itself. For further details on quasi-variational inequalities, we refer to [3-5] and the references therein. In 1980, Giannessi [6] initiated the theory of vector variational inequalities with applications to vector optimization. Since then, it has been growing up in different directions. One of such directions is the application to the theory of vector optimization. However, if the underlying objective function is not differentiable and not convex, then we need to define a nonsmooth vector variational-like inequality by means of Dini directional derivatives or Clarke directional derivatives. For studying such problems by using vector variationallike inequalities, Alshahrani et al. [7], Al-Homidan et al. [8], Ansari and Lee [9], Crespi et al. [10], and Lalitha and Mehta [11] considered a vector variational inequality, defined by means of Dini directional derivatives, called nonsmooth vector variational inequality. The nonsmooth vector optimization is studied in these references by using nonsmooth vector variational inequalities. Motivated by the extension of variational inequalities for vector-valued functions, several researchers started to study the QVI for vector-valued functions, known as vector quasi-variational inequalities (VQVI); see, for example, [12-16] and the references therein. An optimization problem in which the feasible set depends on the solution itself is called quasi-optimization problem [14]. Such problems can be solved by using the vector quasi-variational inequality technique. To the best of our knowledge, no study has been done in the literature to study nonsmooth quasi-variational inequalities which are defined by means of a bifunction, in particular by means of Dini or Clarke directional derivatives. This paper can be treated as the beginning of the study of nonsmooth (vector) quasi-variational inequalities and nonsmooth vector quasioptimization problem.

In this paper, we consider the vector quasi-variationallike inequality problems defined by means of a bifunction and present some existence results for solutions of these problems by using Fan-KKM theorem and a maximal element theorem. By using the technique and methodology adopted in [8], one can easily derive the relations among these inequalities and a vector quasi-optimization problem. Hence, the existence results for a solution of a vector quasi-optimization problem can be derived by using our results. The results of this paper extend several known results in the literature. 


\section{Formulations}

We adopt the following ordering relations. We consider the cones $C=\mathbb{R}_{+}^{\ell}$ and $\stackrel{\circ}{C}:=\operatorname{int} \mathbb{R}_{+}^{\ell}$, where $\mathbb{R}_{+}^{\ell}$ is the nonnegative orthant of $\mathbb{R}^{\ell}$, and $\mathbf{0}$ is the origin of $\mathbb{R}^{\ell}$; let $D$ be a set of $\mathbb{R}_{+}^{\ell}$. Then for all $x, y \in D$,

$$
\begin{aligned}
& x \geq_{C} y \Longleftrightarrow x-y \in C ; \quad x \geq_{C} y \Longleftrightarrow x-y \notin C ;
\end{aligned}
$$

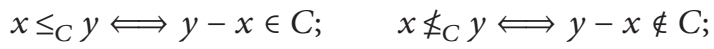

$$
\begin{aligned}
& x \geq_{\mathrm{C}} y \Longleftrightarrow x-y \in \stackrel{\circ}{\mathrm{C}} ; \quad x \underline{ \pm}_{\mathrm{C}} y \Longleftrightarrow x-y \notin \stackrel{\circ}{\mathrm{C}} \text {; }
\end{aligned}
$$

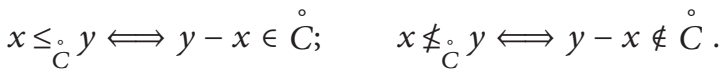

Let $g: \mathbb{R}^{n} \rightarrow \mathbb{R}$ be a real-valued function. The upper Dini directional derivative of $g$ at $x \in K$ in the direction $d \in$ $\mathbb{R}^{n}$ is defined as

$$
g^{D}(x ; d)=\limsup _{t \rightarrow 0^{+}} \frac{g(x+t d)-g(x)}{t} .
$$

For further details on Dini directional derivatives, we refer to the recent book [2].

Let $K$ be a nonempty subset of $\mathbb{R}^{n}, A: K \rightarrow 2^{K}$ a setvalued map, and $\eta: K \times K \rightarrow \mathbb{R}^{n}$ a mapping. Let $h=$ $\left(h_{1}, \ldots, h_{\ell}\right): K \times \mathbb{R}^{n} \rightarrow \mathbb{R}^{\ell}$ be a vector-valued function such that, for each fixed $x \in K, h(x ; d)$ is positively homogeneous in $d$. In particular, we consider $h(x ; d)=f^{D}(x ; d)$ where $f=\left(f_{1}, \ldots, f_{\ell}\right): \mathbb{R}^{n} \rightarrow \mathbb{R}^{\ell}$ a vector-valued function and

$$
f^{D}(x ; d)=\left(f_{1}^{D}(x ; d), \ldots, f_{\ell}^{D}(x ; d)\right) .
$$

The nonsmooth (Stampacchia or Minty type) vector quasi-variational-like inequality problems are defined as follows.

Nonsmooth Stampacchia Vector Quasi-Variational-Like Inequality Problem (NSVQVLIP). Find $\bar{x} \in K$ such that $\bar{x} \in A(\bar{x})$ and

$$
\begin{array}{r}
h(\bar{x} ; \eta(y, \bar{x})) \\
=\left(h_{1}(\bar{x} ; \eta(y, \bar{x})), \ldots, h_{\ell}(\bar{x} ; \eta(y, \bar{x}))\right) \Varangle_{C} \mathbf{0}, \\
\forall y \in A(\bar{x}) .
\end{array}
$$

Minty Vector Quasi-Variational-Like Inequality Problem $(N M V Q V L I P)$. Find $\bar{x} \in K$ such that $\bar{x} \in A(\bar{x})$ and

$$
\begin{array}{r}
h(y ; \eta(\bar{x}, y)) \\
=\left(h_{1}(y ; \eta(\bar{x}, y)), \ldots, h_{\ell}(y ; \eta(\bar{x}, y))\right) \Psi_{C} \mathbf{0}, \\
\forall y \in A(\bar{x}) .
\end{array}
$$

When $A(x)=K$, for all $x \in K$, then these problems were studied in $[8,9,11]$ with applications to vector optimization. Furthermore, if we consider the previous Dini directional derivative as a bifunction $h(x ; d)$, with $x$ referring to a point in $\mathbb{R}^{n}$ and $d$ referring to a direction from $\mathbb{R}^{n}$, that is, if $h(x ; \cdot)=$ $f^{D}(x ; \cdot)$, then the previously mentioned problems are studied in $[7,8,10]$ and the references therein.
The main motivation of this paper is to establish some existence results for solutions of NMVQVLIP and NSVQVLIP by using Fan-KKM theorem or a maximal element theorem. Of course, by using the technique of [8], we can easily establish some results on the relations among NMVQVLIP, NSVQVLIP, and vector quasi-optimization problems [14]. Since the results are straightforward, we are not including them here.

\section{Preliminaries}

Let $K \subseteq \mathbb{R}^{n}$ be a nonempty set. We denote by $\bar{K}$, int $A$, and $\operatorname{co}(K)$ the closure of $K$, the interior of $K$, and the convex hull of $K$, respectively.

Definition 1. Let $K \subseteq \mathbb{R}^{n}$ be a nonempty set and $\eta: K \times K \rightarrow$ $X$ a mapping. The set $K$ is said to be invex with respect to $\eta$ if, for all $x, y \in K$ and all $t \in[0,1]$, we have $x+\operatorname{t\eta }(y, x) \in K$.

We say that the map $\eta$ is skew if, for all $x, y \in K$

$$
\eta(y, x)+\eta(x, y)=\mathbf{0} .
$$

Condition $C$. Let $K \subseteq \mathbb{R}^{n}$ be an invex set with respect to $\eta$ : $K \times K \rightarrow \mathbb{R}^{n}$. Then, for all $x, y \in K, t \in[0,1]$, we have

$$
\eta(x, x+t \eta(y, x))=-t \eta(y, x) .
$$

We adopt the following definition of affineness.

A vector-valued function $g: \mathbb{R}^{n} \rightarrow \mathbb{R}^{\ell}$ is called affine if, for all $x_{1}, x_{2}, \ldots, x_{m} \in \mathbb{R}^{n}$ and $t_{i} \geq 0$ for all $i=1,2, \ldots, m$ with $\sum_{i=1}^{m} t_{i}=1$, we have

$$
g\left(\sum_{i=1}^{m} t_{i} x_{i}\right)=\sum_{i=1}^{m} t_{i} g\left(x_{i}\right) .
$$

The following lemma can be easily proved.

Lemma 2 (see [17]). Let $K$ be a nonempty convex subset of a vector space $X$ and $\eta: K \times K \rightarrow X$ a mapping. If $\eta$ is affine in the first argument and skew, then it is also affine in the second argument.

Definition 3 (see $[18,19]$ ). Let $K \subseteq \mathbb{R}^{n}$ be a nonempty set. A vector-valued function $g: K \rightarrow \mathbb{R}^{\ell}$ is said to be $C$-lower semicontinuous (resp., $C$-upper semicontinuous) at $x \in K$ if for any neighborhood $V$ of $g(x)$, there exists a neighborhood $U$ of $x$ such that $g(y) \in V+C$ for all $y \in U \cap K$ (resp., $g(y) \in V-C$ for all $y \in U \cap K) . g$ is said to be $C$-lower semicontinuous (resp., $C$-upper semicontinuous) on $K$ if it is $C$ lower semicontinuous (resp., C-upper semicontinuous) at every point $x \in K$.

It is shown in [18] that a function $g: K \rightarrow \mathbb{R}^{\ell}$ is $C$ lower semicontinuous if and only if, for all $\alpha \in \mathbb{R}^{\ell}$, the set $\left\{x \in K: g(x) \nsupseteq_{\mathrm{C}} \alpha\right\}$ is closed in $K$.

Definition 4. Let $K \subseteq \mathbb{R}^{n}$ be a nonempty convex set. A vectorvalued function $g: K \rightarrow \mathbb{R}^{\ell}$ is said to be $C$-convex if, for all $x, y \in K$ and all $t \in[0,1]$,

$$
g(t x+(1-t) y) \leq_{C} \operatorname{tg}(x)+(1-t) g(y) .
$$


Definition 5 (see $[18,19]$ ). Let $K \subseteq \mathbb{R}^{n}$ be a nonempty convex set. A vector-valued function $g: K \rightarrow \mathbb{R}^{\ell}$ is said to be $C$ quasiconvex if, for all $\alpha \in \mathbb{R}^{n}$, the set $\left\{x \in K: g(x) \leq_{C} \alpha\right\}$ is convex.

It is shown in [18] that if $g$ is $C$-quasiconvex, then the set $\left\{x \in K: g(x) \leq_{\circ} \alpha\right\}$ is convex.

A vector-valued function $g: \mathbb{R}^{n} \rightarrow \mathbb{R}^{\ell}$ is called positively homogeneous if for all $x \in \mathbb{R}^{n}$ and all $r>0, g(r x)=$ $\operatorname{rg}(x)$.

Definition 6. Let $U$ be a nonempty subset of a topological vector space $E$. A set-valued map $T: U \rightarrow 2^{U}$ is said to be a $K K M$ map provided and $\operatorname{co}(M) \subseteq T(M)=\bigcup_{x \in M} T(x)$ for each finite subset $M$ of $U$, where $\operatorname{co}(M)$ denotes the convex hull of $M$.

The following Fan-KKM theorem [20] will be used in the sequel.

Theorem 7 (see [20]). Let $U$ be a nonempty subset of a Hausdorff topological vector space E. Assume that $T: U \rightarrow$ $2^{U} \backslash\{\emptyset\}$ is a KKM map satisfying the following conditions:

(i) for each $x \in U, T(x)$ is closed;

(ii) for at least one $x \in U, T(x)$ is compact.

Then, $\bigcap_{x \in U} T(x) \neq \emptyset$.

We will use the following maximal element theorem to prove the existence of solutions of nonsmooth vector quasivariational-like inequality problems.

Theorem 8 (see [21, Corollary 3.2]). Let $K$ be a nonempty convex subset of a Hausdorff topological vector space $X$ and $S, T: K \rightarrow 2^{K}$ two set-valued maps. Assume that the following conditions hold:

(i) for all $x \in K, \operatorname{co}(S(x)) \subseteq T(x)$;

(ii) for all $x \in K, x \notin T(x)$ and $S^{-1}(y)=\{x \in K: y \in$ $S(x)$ \} is open in $K$;

(iii) there exist a nonempty compact convex subset $D \subseteq K$ and a nonempty compact subset $B$ of $K$ such that for each $x \in K \backslash B$, there exists $\tilde{y} \in D$ such that $x \in$ $\operatorname{int} S^{-1}(\tilde{y})$.

Then, there exists $\bar{x} \in K$ such that $S(\bar{x})=\emptyset$.

\section{Existence Results}

Definition 9 (see [8]). Let $K \subseteq \mathbb{R}^{n}$ be a nonempty set and $\eta: K \times K \rightarrow \mathbb{R}^{n}$ a mapping. A vector-valued bifunction $h=\left(h_{1}, \ldots, h_{\ell}\right): K \times \mathbb{R}^{n} \rightarrow \mathbb{R}^{\ell}$ is said to be

(a) C-pseudomonotone with respect to $\eta$ on $K$ if, for all $x, y \in K$,

$$
\begin{aligned}
& h(x ; \eta(y, x)) \varliminf_{C} \mathbf{0} \\
& \quad \text { implies } h(y ; \eta(x, y)) \nsupseteq_{C} \mathbf{0},
\end{aligned}
$$

(b) C-properly subodd if

$$
h\left(x ; d_{1}\right)+h\left(x ; d_{2}\right)+\cdots+h\left(x ; d_{m}\right) \geq_{C} \mathbf{0},
$$

for every $d_{i} \in \mathbb{R}^{n}, i=1,2, \ldots, m$ with $\sum_{i=1}^{m} d_{i}=\mathbf{0}$ and $x \in K$.

The definition of proper suboddness is considered in [11]. Of course, if $m=2$, the definition of proper suboddness reduces to the definition of suboddness.

Definition 10. Let $K$ be a nonempty convex subset of $\mathbb{R}^{n}$. A function $g: K \rightarrow \mathbb{R}^{\ell}$ is said to be hemicontinuous if, for all $x, y \in K$, the mapping $t \mapsto g(y+t(x-y))$ is continuous. The upper and lower hemicontinuity can be defined analogously.

Definition 11. Let $K \subseteq \mathbb{R}^{n}$ be an invex set with respect to $\eta: K \times K \rightarrow \mathbb{R}^{n}$. A function $g: K \rightarrow \mathbb{R}^{\ell}$ is said to be $\eta$-hemicontinuous if, for all $x, y \in K$, the mapping $t \mapsto g(y+t \eta(x, y))$ is continuous. The upper and lower $\eta$ hemicontinuity can be defined analogously.

The following concept of $\eta$-upper sign continuity for the bifunction $h$ is considered in [8].

Definition 12 (see [8]). Let $K \subseteq \mathbb{R}^{n}$ be a nonempty invex set with respect to $\eta: K \times K \rightarrow \mathbb{R}^{n}$. A vector-valued bifunction $h=\left(h_{1}, \ldots, h_{\ell}\right): K \times \mathbb{R}^{n} \rightarrow \mathbb{R}^{\ell}$ is said to be $\eta$-upper sign continuous if, for all $x, y \in K$ and $t \in(0,1)$,

$$
\begin{aligned}
h(x+t \eta(y, x) ; \eta(y, x)) & ⿱_{\circ} \mathbf{0} \\
\text { implies } h(x ; \eta(y, x)) & \varliminf_{C} \mathbf{0} .
\end{aligned}
$$

Remark 13. It can be easily seen that if $\eta$ is skew and $h$ is $\eta$-upper hemicontinuous in the first argument, then it is $\eta$ upper sign continuous, but the converse is not true in general.

The following result provides the relations between NSVQVLIP and NMVQVLIP when the set-valued map $A$ : $K \rightarrow 2^{K}$ is invex valued.

Proposition 14. Let $K \subseteq \mathbb{R}^{n}$ be a nonempty invex set with respect to $\eta: K \times K \rightarrow \mathbb{R}^{n}$ such that Condition $C$ holds. Let $A: K \rightarrow 2^{K}$ be a set-valued map such that, for each $x \in$ $K, A(x)$ is a nonempty and invex set with respect to $\eta$. Let the vector-valued bifunction $h: K \times \mathbb{R}^{n} \rightarrow \mathbb{R}^{\ell}$ be C-properly subodd, $C$-pseudomonotone with respect to $\eta$, and $\eta$-upper sign continuous such that, for each fixed $x \in K, h(x ; \cdot)$ is positively homogeneous. Then, $\bar{x} \in K$ is a solution of NSVQVLIP if and only if it is a solution of NMVQVLIP.

Proof. It is similar to the proof of Proposition 7.7 in [8]. However, we include it for the sake of completeness of the paper.

The $C$-pseudomonotonicity of $h$ with respect to $\eta$ implies that every solution of NSVQVLIP is a solution of NMVQVLIP. 
Conversely, let $\bar{x} \in K$ be a solution of NMVQVLIP. Then, $\bar{x} \in A(\bar{x})$, and

$$
h(y ; \eta(\bar{x}, y)){\beth_{\mathrm{C}}}_{0} \mathbf{0}, \quad \forall y \in A(\bar{x}) .
$$

Since $A(\bar{x})$ is invex, we have $y_{t}=\bar{x}+t \eta(y, \bar{x}) \in A(\bar{x})$ for all $t \in(0,1)$, and therefore, (13) becomes

$$
h\left(y_{t} ; \eta\left(\bar{x}, y_{t}\right)\right) \ddagger_{\mathrm{C}} \mathbf{0} .
$$

By Condition C, $\eta\left(\bar{x}, y_{t}\right)=-t \eta(y, \bar{x})$, and thus,

$$
h\left(y_{t} ;-t \eta(y, \bar{x})\right) \ddagger_{C} \mathbf{0} .
$$

By positive homogeneity and $C$-proper suboddness of $h$, we have

$$
h\left(y_{t} ; \eta(y, \bar{x})\right){\ddagger_{\mathrm{C}}}_{\mathbf{0}} .
$$

Thus, the $\eta$-upper sign continuity of $h$ yields $\bar{x} \in K$ is a solution of NSVQVLIP.

The following result gives the equivalence between NSVQVLIP and NMVQVLIP when the set-valued map $A$ : $K \rightarrow 2^{K}$ is convex valued.

Proposition 15. Let $K \subseteq \mathbb{R}^{n}$ be a nonempty convex set, and let $\eta: K \times K \rightarrow \mathbb{R}^{n}$ be affine in the first argument and skew. Let $A: K \rightarrow 2^{K}$ be a set-valued map such that, for each $x \in K, A(x)$ is a nonempty and convex set. Let the vector-valued bifunction $h: K \times \mathbb{R}^{n} \rightarrow \mathbb{R}^{\ell}$ be C-properly subodd, C-pseudomonotone with respect to $\eta$, and $\eta$-upper sign continuous such that, for each fixed $x \in K, h(x ; \cdot)$ is positively homogeneous. Then, $\bar{x} \in K$ is a solution of NSVQVLIP if and only if it is a solution of NMVQVLIP.

Proof. It is similar to the proof of Proposition 7.8 in [8]. However, we include it for the sake of completeness of the paper.

The C-pseudomonotonicity of $h$ with respect to $\eta$ implies that every solution of NSVQVLIP is a solution of NMVQVLIP.

Conversely, let $\bar{x} \in K$ be a solution of NMVQVLIP. Then, $\bar{x} \in A(\bar{x})$, and

$$
h(y ; \eta(\bar{x}, y)){\beth_{\mathrm{C}}}_{0} \mathbf{0}, \quad \forall y \in A(\bar{x}) .
$$

Since $A(\bar{x})$ is convex, we have $y_{t}=\bar{x}+t(y-\bar{x}) \in A(\bar{x})$ for all $t \in(0,1)$, and therefore, (17) becomes

$$
h\left(y_{t} ; \eta\left(\bar{x}, y_{t}\right)\right) \pm_{\mathrm{C}} \mathbf{0} .
$$

Since $\eta$ is affine in the first argument and skew, by Lemma 2 , $\eta$ is also affine in the second argument. Since $\eta(x, x)=\mathbf{0}$ by skewness of $\eta$, we obtain

$$
\begin{aligned}
h\left(y_{t} ; \eta\left(\bar{x}, y_{t}\right)\right) & =h\left(y_{t} ; \eta \eta(\bar{x}, y)+(1-t) \eta(\bar{x}, \bar{x})\right) \\
& =h\left(y_{t} ; \eta(\bar{x}, y)\right) \ddagger_{C} \mathbf{0} .
\end{aligned}
$$

By positive homogeneity of $h$ in the second argument, we have

$$
h\left(y_{t} ; \eta(\bar{x}, y)\right) ఖ_{C} \mathbf{0} .
$$

Since $\eta(y, \bar{x})+\eta(\bar{x}, y)=\mathbf{0}$ by skewness of $\eta$, the $C$-proper suboddness of $h$ implies that

$$
h\left(y_{t} ; \eta(y, \bar{x})\right) \not_{C} \mathbf{0} .
$$

The $\eta$-upper sign continuity of $h$ yields $\bar{x} \in K$ is a solution of NSVQVLIP.

Throughout the rest of the paper, unless otherwise specified, we assume that $A: K \rightarrow 2^{K}$ is a set-valued map such that $A(x)$ is nonempty convex for all $x \in K, A^{-1}(y)$ is open for all $y \in K$, and the set $\mathscr{F}=\{x \in K: x \in A(x)\}$ is closed.

We present some existence results for the solutions of NSVQVLIP and NMVQVLIP without boundedness assumption on the underlying set $K$.

Theorem 16. Let $K \subseteq \mathbb{R}^{n}$ be a nonempty convex set, and $\eta: K \times K \rightarrow \mathbb{R}^{n}$ be skew, affine, and lower semicontinuous in the first argument. Let $h=\left(h_{1}, \ldots, h_{\ell}\right): K \times \mathbb{R}^{n} \rightarrow \mathbb{R}^{\ell}$ be C-properly subodd, positively homogeneous in the second argument, and C-pseudomonotone with respect to $\eta$ such that $x \mapsto h(y ; \eta(x, y))$ is continuous. Assume that there exist a nonempty compact convex subset $D$ of $K$ and $\tilde{y} \in D$ such that, for all $x \in K \backslash D, \tilde{y} \in A(x)$ and $h(x ; \eta(\tilde{y}, x)) \leq_{\mathrm{C}} 0$ Then, there exists a solution $\bar{x} \in K$ of MVQVLIP.

Furthermore, if $h$ is $\eta$-upper sign continuous, then $\bar{x} \in K$ is a solution of SVQVLIP.

Proof. For all $x \in K$, we define two set-valued maps $S_{1}, S_{2}$ : $K \rightarrow 2^{K}$ by

$$
\begin{aligned}
& S_{1}(x)=\left\{y \in K: h(x ; \eta(y, x)) \leq_{\mathrm{C}} \mathbf{0}\right\}, \\
& S_{2}(x)=\left\{y \in K: h(y ; \eta(x, y)) \geq_{{ }_{C}} \mathbf{0}\right\} .
\end{aligned}
$$

For all $x, y \in K$ and for each $i=1,2$, we also define other two set-valued maps $P_{i}: K \rightarrow 2^{K}$ and $Q_{i}: K \rightarrow 2^{K}$ by

$$
\begin{aligned}
& P_{i}(x)= \begin{cases}A(x) \cap S_{i}(x), & \text { if } x \in \mathscr{F}, \\
A(x), & \text { if } x \in K \backslash \mathscr{F},\end{cases} \\
& Q_{i}(y)=K \backslash P_{i}^{-1}(y) .
\end{aligned}
$$

For each $i=1,2$ and for all $y \in K$, we have (see, e.g., [22])

$$
P_{i}^{-1}(y)=\left[(K \backslash \mathscr{F}) \cup S_{i}^{-1}(y)\right] \cap A^{-1}(y),
$$

and therefore,

$$
Q_{i}(y)=\left[\mathscr{F} \cap\left(K \backslash S_{i}^{-1}(y)\right)\right] \cup\left[K \backslash A^{-1}(y)\right] .
$$

The rest of the proof is divided into the following four steps.

(a) We claim that $Q_{1}$ is a KKM map on $K$. 
Assume the contrary that $Q_{1}$ is not a KKM map. Then, there exist a finite set $\left\{y_{1}, \ldots, y_{m}\right\}$ in $K$ and $t_{1}, \ldots, t_{m} \geq 0$ with $\sum_{i=1}^{m} t_{i}=1$ such that $\hat{x}=\sum_{i=1}^{m} t_{i} y_{i} \notin Q_{1}\left(y_{i}\right)$ for all $i=$ $1, \ldots, m$; that is,

$$
\widehat{x} \in P_{1}^{-1}\left(y_{i}\right) \Longleftrightarrow y_{i} \in P_{1}(\widehat{x}) \quad \forall i=1, \ldots, m .
$$

If $\widehat{x} \in \mathscr{F}$, then $P_{1}(\widehat{x})=A(\widehat{x}) \cap S_{1}(\widehat{x})$, and therefore,

$$
y_{i} \in S_{1}(\widehat{x}), \quad y_{i} \in A(\widehat{x}) \quad \forall i=1, \ldots, m .
$$

Hence,

$$
h\left(\widehat{x} ; \eta\left(y_{i}, \widehat{x}\right)\right) \leq_{\mathrm{C}} \mathbf{0}, \quad y_{i} \in A(\widehat{x}) \quad \forall i=1, \ldots, m .
$$

Since $\stackrel{\circ}{C}$ is a convex cone and $t_{i} \geq 0$ with $\sum_{i=1}^{m} t_{i}=1$, we have

$$
\sum_{i=1}^{m} t_{i} h\left(\widehat{x} ; \eta\left(y_{i}, \widehat{x}\right)\right) \leq_{\mathrm{C}} \mathbf{0} .
$$

Since $\eta$ is skew, we have $\eta(x, x)=\mathbf{0}$. By the affineness of $\eta$ in the first argument, we have

$$
\sum_{i=1}^{m} t_{i} \eta\left(y_{i}, \widehat{x}\right)=\eta\left(\sum_{i=1}^{m} t_{i} y_{i}, \widehat{x}\right)=\eta(\widehat{x}, \widehat{x})=\mathbf{0} .
$$

Since $h$ is $C$-proper subodd, we have

$$
\sum_{i=1}^{m} h\left(\widehat{x} ; t_{i} \eta\left(y_{i}, \widehat{x}\right)\right) \geq_{C} \mathbf{0} .
$$

By positive homogeneity of $h$, we obtain

$$
\sum_{i=1}^{m} t_{i} h\left(\widehat{x} ; \eta\left(y_{i}, \widehat{x}\right)\right) \geq_{C} \mathbf{0},
$$

a contradiction of (29).

If $\widehat{x} \in K \backslash \mathscr{F}$, then $\widehat{x} \notin A(\widehat{x})$. By the definition of $Q_{1}$, we have $P_{1}(\widehat{x})=A(\widehat{x})$, and therefore, $y_{i} \in P_{1}(\widehat{x})=A(\widehat{x})$ for all $i=1, \ldots, m$. Since $A(\widehat{x})$ is convex, we obtain $\widehat{x} \in A(\widehat{x})$, again a contradiction. Hence, $Q_{1}$ is a KKM map.

(b) We show that $Q_{1}(\tilde{y})=K \backslash P_{1}^{-1}(\tilde{y}) \subseteq D$, where $\tilde{y}$ and $D$ are the same as in the hypothesis.

Indeed, if $x \in Q_{1}(\tilde{y}) \backslash D$, then $x \in\left[\mathscr{F} \cap\left(K \backslash S_{1}^{-1}(\tilde{y})\right)\right] \cup[K \backslash$ $\left.A^{-1}(\tilde{y})\right]$; that is, either $x \in \mathscr{F} \cap\left(K \backslash S_{1}^{-1}(\tilde{y})\right)$ or $x \in K \backslash A^{-1}(\tilde{y})$.

If $x \in \mathscr{F} \cap\left(K \backslash S_{1}^{-1}(\tilde{y})\right)$, then $x \in \mathscr{F}$ and $x \in K \backslash S_{1}^{-1}(\tilde{y})$; that is, $x \in A(x)$ and $h(x ; \eta(\tilde{y}, x)) \not_{\mathrm{C}} \mathbf{0}$, a contradiction to our assumption that $h(x ; \eta(\tilde{y}, x)) \leq_{\mathrm{C}} \mathbf{0}$.

If $x \in K \backslash A^{-1}(\tilde{y})$, then $x \notin A^{-1}(\tilde{y})$ if and only if $\tilde{y} \notin$ $A(x)$, again a contradiction to our assumption that $\tilde{y} \in A(x)$. Hence, $Q_{1}(\tilde{y}) \subseteq D$.

(c) We show that $\bigcap_{y \in K} \overline{Q_{1}(y)} \neq \emptyset$.

Since $D$ is compact, $\overline{Q_{1}(\tilde{y})}$ is also compact. Moreover, since $Q_{1}$ is a KKM map,

$$
\operatorname{co}\left\{y_{1}, \ldots, y_{m}\right\} \subseteq \bigcup_{i=1}^{m} Q_{1}\left(y_{i}\right) \subseteq \bigcup_{i=1}^{m} \overline{Q_{1}\left(y_{i}\right)},
$$

for each finite subset $\left\{y_{1}, \ldots, y_{m}\right\}$ of $K$. Then by Theorem 7 , we get $\bigcap_{y \in K} \overline{Q_{1}(y)} \neq \emptyset$. (d) Next, we claim that $\bigcap_{y \in K} \overline{Q_{1}(y)} \subseteq \bigcap_{y \in K} Q_{2}(y)$.

Let $z \in \bigcap_{y \in K} \overline{Q_{1}(y)}$; then $z \in \overline{Q_{1}(y)}$ for each $y \in K$. For an arbitrary element $y \in K$, we have to show that $z \in Q_{2}(y)$.

Since $z \in \overline{Q_{1}(y)}$, there exists a sequence $\left\{z_{m}\right\} \subseteq Q_{1}(y)$ such that $\left\{z_{m}\right\}$ converges to $z$. Since $\left\{z_{m}\right\} \subseteq Q_{1}(y)$, we have

$$
\left\{z_{m}\right\} \subseteq K \backslash P_{1}^{-1}(y)=\left[\mathscr{F} \cap\left(K \backslash S_{1}^{-1}(y)\right)\right] \cup\left[K \backslash A^{-1}(y)\right] .
$$

Then, either $\left\{z_{m}\right\} \subseteq \mathscr{F} \cap\left(K \backslash S_{1}^{-1}(y)\right)$ or $\left\{z_{m}\right\} \subseteq K \backslash A^{-1}(y)$.

If $\left\{z_{m}\right\} \subseteq \mathscr{F} \cap\left(K \backslash S_{1}^{-1}(y)\right)$, then $\left\{z_{m}\right\} \subseteq \mathscr{F}$ and $\left\{z_{m}\right\} \subseteq$ $K \backslash S_{1}^{-1}(y)$. It follows that $\left\{z_{m}\right\} \subseteq \mathscr{F}$ and $h\left(z_{m} ; \eta\left(y, z_{m}\right)\right) \not_{\mathrm{C}} \mathbf{0}$. Since $\mathscr{F}$ is closed and $z_{m} \rightarrow z$, we have $z \in \mathscr{F}$; that is, $z \in$ $A(z)$. By $C$-pseudomonotonicity of $h$, we obtain

$$
z \in A(z), \quad h\left(y ; \eta\left(z_{n}, y\right)\right) \geq \mathbf{0} .
$$

By the continuity of $x \mapsto h(y ; \eta(x, y)) h$, we get $h(y, \eta(z, y)) \ddagger_{C} 0$. This implies that $z \in A(z)$ and $y \notin S_{2}(z)$; that is, $z \notin(K \backslash \mathscr{F}) \cup S_{2}^{-1}(y)$, and hence, $z \notin P_{2}^{-1}(y)$. Therefore, $z \in A(z)$, and $z \in K \backslash P_{2}^{-1}(y)=Q_{2}(y)$.

Let $\left\{z_{m}\right\} \subseteq K \backslash A^{-1}(y)$. Since $A^{-1}(y)$ is open in $K$, for all $y \in K, K \backslash A^{-1}(y)$ is closed in $K$. Since $z_{m} \rightarrow z$, we have $z \in K \backslash A^{-1}(y)$. Hence, $z \notin A^{-1}(y) \Leftrightarrow y \notin A(z)$, which implies that

$$
\begin{aligned}
y \notin P_{2}(z) & \Longleftrightarrow z \notin P_{2}^{-1}(y) \\
& \Longleftrightarrow z \in K \backslash P_{2}^{-1}(y) \Longleftrightarrow z \in Q_{2}(y) .
\end{aligned}
$$

From (c), we get $\bigcap_{y \in K} Q_{2}(y) \neq \emptyset$. Hence, there exists $\bar{x} \in$ $K$ such that

$$
\bar{x} \in \bigcap_{y \in K}\left[K \backslash P_{2}^{-1}(y)\right]=K \backslash \bigcup_{y \in K} P_{2}^{-1}(y) .
$$

This implies that $P_{2}(\bar{x})=\emptyset$.

If $\bar{x} \in K \backslash \mathscr{F}$, then $P_{2}(\bar{x})=A(\bar{x})=\emptyset$, a contradiction. Otherwise, $\bar{x} \in \mathscr{F}$; then $\emptyset=P_{2}(\bar{x})=A(\bar{x}) \cap S_{2}(\bar{x})$. Therefore, $\bar{x} \in A(\bar{x})$ such that $h(y ; \eta(\bar{x}, y)) \nsupseteq_{C_{0}} \mathbf{0}$ for all $y \in A(\bar{x})$. From Proposition $15, \bar{x} \in K$ is a solution of SVQVLIP.

Remark 17. (a) Theorem 16 extends and generalizes [8, Theorem 7.34], [9, Theorem 5.1], and [11, Theorem 2.2].

(b) If $A$ is a closed map, then the set $\mathscr{F}=\{x \in K: x \in$ $A(x)\}$ is closed.

By using maximal element Theorem 8 , we present the following existence result for solutions of NSVQVLIP and NMVQVLIP without boundedness assumption on the underlying set.

Theorem 18. Let $K \subseteq \mathbb{R}^{n}$ be a nonempty convex set, and let $\eta: K \times K \rightarrow \mathbb{R}$ be skew. Let $h: K \times \mathbb{R}^{n} \rightarrow \mathbb{R}^{\ell}$ be $C$ pseudomonotone with respect to $\eta$ such that $h(x ; \mathbf{0})=\mathbf{0}$ for all $x \in K$; the set $M=\left\{y \in K: h(x, \eta(y, x)) \leq_{0} \mathbf{0}\right\}$ is convex, and the set $L=\left\{x \in K: h(y, \eta(x, y)) \nsupseteq_{\mathrm{C}} \mathbf{0}\right\}$ is closed in K. Assume that there exist a nonempty compact convex subset $D \subseteq K$ and 
a nonempty compact subset $B$ of $K$ such that, for each $x \in K \backslash B$, there exists $\tilde{y} \in D$ such that $\tilde{y} \in A(x)$ and $h(\tilde{y} ; \eta(x, \tilde{y})) \geq_{C} \mathbf{0}$. Then, there exists a solution $\bar{x} \in K$ of NMVQVLIP.

Furthermore, if $h$ is C-properly subodd, $\eta$-upper sign continuous, and for each fixed $x \in K, h(x ; \cdot)$ is positively homogeneous, then $\bar{x} \in K$ is a solution of NSVQVLIP.

Proof. For each $x \in K$, define two set-valued maps $P, Q$ : $K \rightarrow 2^{K}$ by

$$
\begin{aligned}
& P(x)=\left\{y \in K: h(y ; \eta(x, y)) \geq_{C} \mathbf{0}\right\}, \\
& Q(x)=\left\{y \in K: h(x ; \eta(y, x)) \leq_{C} \mathbf{0}\right\} .
\end{aligned}
$$

Then, $x \notin Q(x)$ for all $x \in K$. Indeed, by skewness of $\eta$, $\eta(x, x)=\mathbf{0}$ for all $x \in K$. By assumption, $\mathbf{0}=h(x ; \eta(x, x))=$ $h(x ; \mathbf{0})=\mathbf{0} \Varangle_{\mathrm{C}} \mathbf{0}$. Thus, $x \notin Q(x)$.

By hypothesis, the complement of $P^{-1}(y)$ in $K$,

$$
\left[P^{-1}(y)\right]^{c}=\left\{x \in K: h(y, \eta(x, y)) \Psi_{C} \mathbf{0}\right\},
$$

is closed in $K$ for each $y \in K$. Therefore, $P^{-1}(y)$ is open in $K$ for all $y \in K$.

Define other two set-valued maps $S, T: K \rightarrow 2^{K}$ by

$$
\begin{aligned}
& S(x)= \begin{cases}A(x) \cap P(x), & \text { if } x \in \mathscr{F}, \\
A(x), & \text { if } x \in K \backslash \mathscr{F},\end{cases} \\
& T(x)= \begin{cases}A(x) \cap Q(x), & \text { if } x \in \mathscr{F}, \\
A(x), & \text { if } x \in K \backslash \mathscr{F} .\end{cases}
\end{aligned}
$$

Since, for all $x \in K, x \notin Q(x)$, we have $x \notin T(x)$.

By $C$-pseudomonotonicity of $h$, we have $P(x) \subseteq Q(x)$ for all $x \in K$. Since $A(x)$ and $Q(x)$ are convex, for all $x \in K$, we have

$$
S(x) \subseteq T(x) \Longrightarrow \operatorname{co}(S(x)) \subseteq \operatorname{co}(T(x))=T(x) .
$$

Since, for each $y \in K, A^{-1}(y)$ and $P^{-1}(y)$ are open in $K$, $(A \cap P)^{-1}(y)=A^{-1}(y) \cap P^{-1}(y)$ is open in $K$. Also, since, for each $y \in K$,

$$
S^{-1}(y)=\left(A^{-1}(y) \cap P^{-1}(y)\right) \cup\left((K \backslash \mathscr{F}) \cap A^{-1}(y)\right)
$$

(see, e.g., the proof of [23, Lemma 2.3]) and $K \backslash \mathscr{F}$ is open in $K$, we have that $S^{-1}(y)$ is open in $K$. Therefore, by Theorem 8 , there exists $\bar{x} \in K$ such that $S(\bar{x})=\emptyset$. If $\bar{x} \in K \backslash \mathscr{F}$, then $A(\bar{x})=\emptyset$, a contradiction to our assumption. So, $\bar{x} \in \mathscr{F}$, and thus, $A(\bar{x}) \cap P(\bar{x})=\emptyset$. Therefore,

$$
\bar{x} \in A(\bar{x}), \quad h(y ; \eta(\bar{x}, y)) \Psi_{C} \mathbf{0}, \quad \forall y \in A(\bar{x}) .
$$

Thus, $\bar{x}$ is a solution of NMVQVLIP.

By Proposition $15, \bar{x} \in K$ is a solution of NSVQVLIP.

Remark 19. If, for each fixed $x \in K$, the vector-valued function $y \mapsto h(x, \eta(y, x))$ is $C$-quasiconvex, then the set $M=\left\{y \in K: h(x, \eta(y, x)) \leq_{\mathrm{C}} \mathbf{0}\right\}$ is convex.
Remark 20. For all $x \in K$, the set $M=\{y \in K$ : $\left.h(x, \eta(y, x)) \leq_{\mathrm{C}} \mathbf{0}\right\}$ is convex, if $\eta$ is affine in the first argument and $h$ is $C$-convex in the second argument.

Indeed, let $y_{1}, y_{2} \in M$. Since $\stackrel{\circ}{C}$ is a convex cone, for all $t \in(0,1)$, we have

$$
\operatorname{th}\left(x ; \eta\left(y_{1}, x\right)\right) \leq_{\mathrm{C}} \mathbf{0}, \quad(1-t) h\left(x ; \eta\left(y_{2}, x\right)\right) \leq_{\mathrm{C}} \mathbf{0 .}
$$

By adding these relations, we get

$$
\operatorname{th}\left(x ; \eta\left(y_{1}, x\right)\right)+(1-t) h\left(x ; \eta\left(y_{2}, x\right)\right) \leq_{C} \mathbf{0} .
$$

Since $h$ is $C$-convex in the second argument, we have

$$
\begin{aligned}
& h\left(x ; \operatorname{t\eta }\left(y_{1}, x\right)+(1-t) \eta\left(y_{2}, x\right)\right) \\
& \quad \leq_{C} \operatorname{th}\left(x ; \eta\left(y_{1}, x\right)\right)+(1-t) h\left(x ; \eta\left(y_{2}, x\right)\right) .
\end{aligned}
$$

By combining relations (45) and (46), we obtain

$$
h\left(x ; \operatorname{t\eta }\left(y_{1}, x\right)+(1-t) \eta\left(y_{2}, x\right)\right) \leq_{C} \mathbf{0 .}
$$

Since $\eta$ is affine in the first argument, we get

$$
h\left(x ; \eta\left(t y_{1}+(1-t) y_{2}, x\right)\right) \leq_{\mathrm{C}} \mathbf{0},
$$

and hence, $t y_{1}+(1-t) y_{2} \in M$. Thus, for all $x \in K, M$ is a convex set.

Remark 21. The set $L=\left\{x \in K: h(y, \eta(x, y)) \Psi_{C} \mathbf{0}\right\}$ is closed in $K$ if the vector-valued function $x \mapsto h(y, \eta(x, y))$ is $C$ lower semicontinuous for each fixed $y \in K$.

\section{Conclusions}

In this paper, we defined vector quasi-variational-like inequality problems by means of a bifunction and established two existence results for solutions of these problems. One can treat upper Dini directional derivative as a bifunction, and hence, the bifunction can be replaced by upper Dini directional derivative. Then, we get the so-called nonsmooth vector quasi-variational-like inequality problem. By using the technique and methodology given in [8], one can easily derive some relations between vector quasi-variational-like inequality problems and a vector quasi-optimization problem. Since the results are straightforward, we have not included them in this paper. Of course, the results of this paper extend several known results in the literature, namely, [9, Theorem 5.1], [11, Theorem 3.1], and [8, Theorem 7.34] from vector variational (-like) inequality problems to vector quasi-variational (-like) inequality problems. This paper can be treated as the first in this direction.

\section{Acknowledgments}

This research was done during the visit of the second author to King Fahd University of Petroleum and Minerals, Dhahran, Saudi Arabia, and it was supported by KFUPM Funded Research Project no. IN121035. 


\section{References}

[1] A. Bensoussan, M. Goursat, and J. L. Lions, "Contrôle impulsionnel et inéquations quasi-variationnelles stationnaires," Comptes Rendus Mathematique, vol. 276, pp. A1279-A1284, 1973.

[2] Q. H. Ansari, C. S. Lalitha, and M. Mehta, Generalized Convexity, Nonsmooth Variational Inequalities and Nonsmooth Optimization, CRC Press, Taylor and Francis Group, London, UK, 2014.

[3] C. Baiocchi and A. Capelo, Variational and Quasivariational Inequalities, John Wiley \& Sons, New York, NY, USA, 1984.

[4] A. Bensoussan and J. L. Lions, Applications des Inéquations Variationnelles en Contrôle Stochastique, Dunod, Paris, France, 1978.

[5] A. S. Kravchuk and P. J. Neittaanmäki, Variational and Quasi Variational Inequalities in Mechanics, Springer, Dordrecht, The Netherlands, 2007.

[6] F. Giannessi, "Theorems of alternative, quadratic programs and complementarity problems," in Variational Inequalities and Complementarity Problems, R. W. Cottle, F. Giannessi, and J. L. Lions, Eds., pp. 151-186, Wiley, Chichester, UK, 1980.

[7] M. Alshahrani, Q. H. Ansari, and S. Al-Homidan, "Nonsmooth variational-like inequalities and nonsmooth vector optimization," Optimization Letters, 2013.

[8] S. Al-Homidan, Q. H. Ansari, and J. C. Yao, "Nonsmooth invexities, invariant monotonicities and nonsmooth vector variational inequalities with applications to vector optimization," in Adances in Vector Optimization, Q. H. Ansari and J. C. Yao, Eds., Springer, Berlin, Germany, 2012.

[9] Q. H. Ansari and G. M. Lee, "Nonsmooth vector optimization problems and Minty vector variational inequalities," Journal of Optimization Theory and Applications, vol. 145, no. 1, pp. 1-16, 2010.

[10] G. P. Crespi, I. Ginchev, and M. Rocca, "Some remarks on the Minty vector variational principle," Journal of Mathematical Analysis and Applications, vol. 345, no. 1, pp. 165-175, 2008.

[11] C. S. Lalitha and M. Mehta, "Vector variational inequalities with cone-pseudomonotone bifunctions," Optimization, vol. 54, no. 3, pp. 327-338, 2005.

[12] Q. H. Ansari, W. K. Chan, and X. Q. Yang, "The system of vector quasi-equilibrium problems with applications," Journal of Global Optimization, vol. 29, no. 1, pp. 45-57, 2004.

[13] Q. H. Ansari, W. K. Chan, and X. Q. Yang, "Weighted quasivariational inequalities and constrained Nash equilibrium problems," Taiwanese Journal of Mathematics, vol. 10, no. 2, pp. 361-380, 2006.

[14] Q. H. Ansari and J. C. Yao, "On vector quasi-equilibrium problems," in Equilibrium Problems and Variational Models, $\mathrm{P}$. Daniele, F. Giannessi, and A. Maugeri, Eds., pp. 1-18, Kluwer Academic Publishers, London, UK, 2003.

[15] F. Giannessi, Vector Variational Inequalities and Vector Equilibria, Mathematical Theories, Kluwer Academic Publishers, Dordrecht, The Netherlands, 2000.

[16] G. X. Z. Yuan, KKM Theory and Applications in Nonlinear Analysis, Marcel Dekker, New York, NY, USA, 1999.

[17] J. W. Peng, "Criteria for generalized invex monotonicities without Condition C," European Journal of Operational Research, vol. 170, no. 2, pp. 667-671, 2006.

[18] M. Bianchi, N. Hadjisavvas, and S. Schaible, "Vector equilibrium problems with generalized monotone bifunctions," Journal of Optimization Theory and Applications, vol. 92, no. 3, pp. 527-542, 1997.
[19] D. T. Luc, Theory of Vector Optimization, vol. 319 of Lecture Notes in Economics and Mathematical Systems, Springer, Berlin, Germany, 1989.

[20] K. Fan, "A generalization of Tychonoff's fixed point theorem," Mathematische Annalen, vol. 142, pp. 305-310, 1961.

[21] L. J. Lin, Z. T. Yu, Q. H. Ansari, and L. P. Lai, "Fixed point and maximal element theorems with applications to abstract economies and minimax inequalities," Journal of Mathematical Analysis and Applications, vol. 284, no. 2, pp. 656-671, 2003.

[22] P. Q. Khanh and L. M. Luu, "Some existence results for vector quasivariational inequalities involving multifunctions and applications to traffic equilibrium problems," Journal of Global Optimization, vol. 32, no. 4, pp. 551-568, 2005.

[23] X. P. Ding, "Existence of solutions for quasi-equilibrium problems in noncompact topological spaces," Computers \& Mathematics with Applications, vol. 39, no. 3-4, pp. 13-21, 2000. 


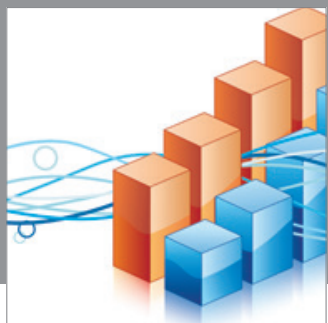

Advances in

Operations Research

mansans

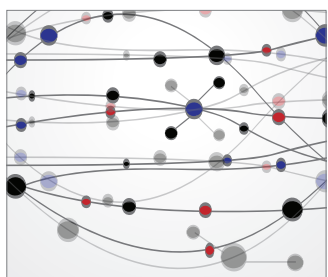

The Scientific World Journal
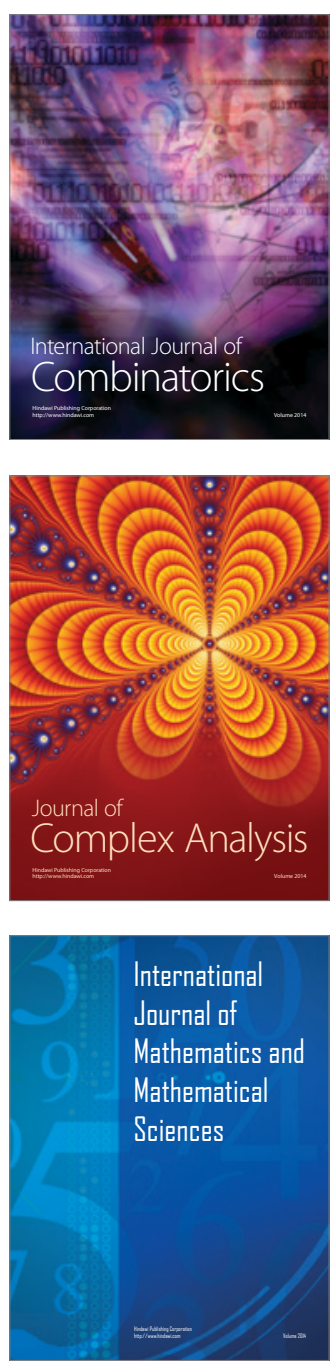
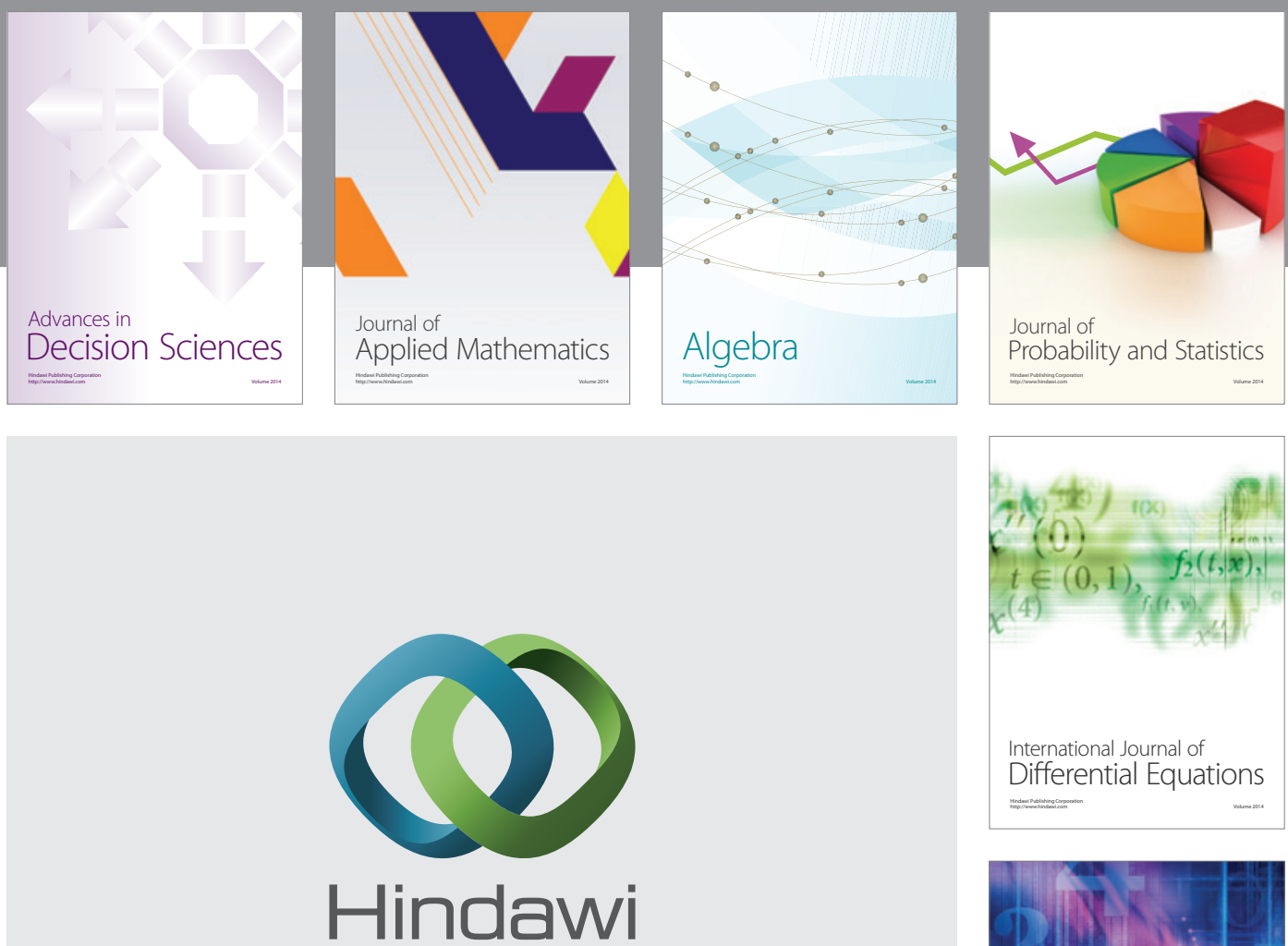

Submit your manuscripts at http://www.hindawi.com
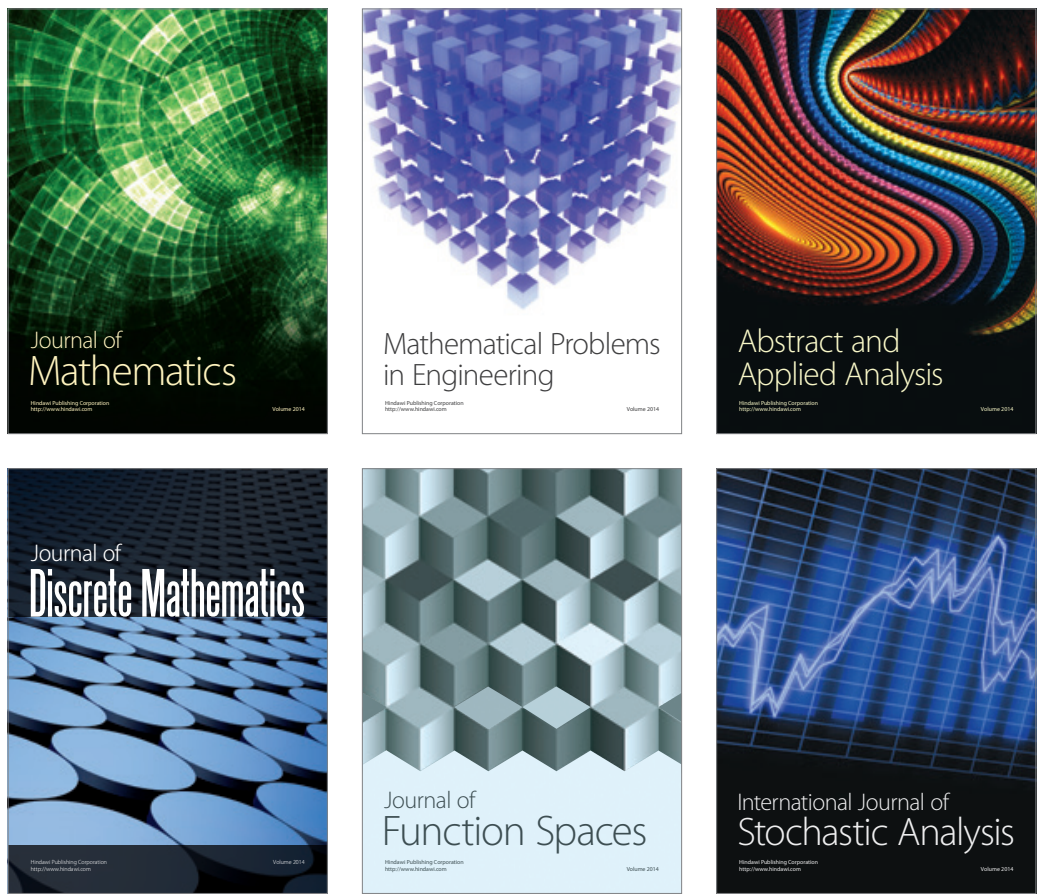

Journal of

Function Spaces

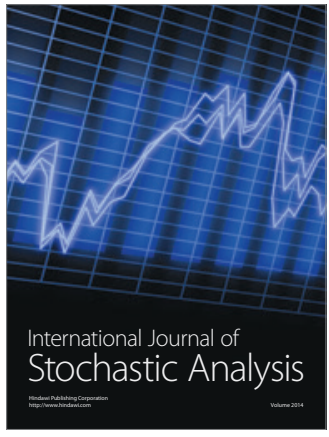

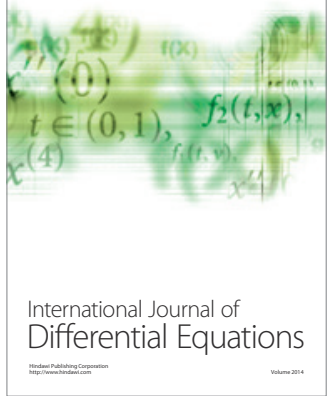
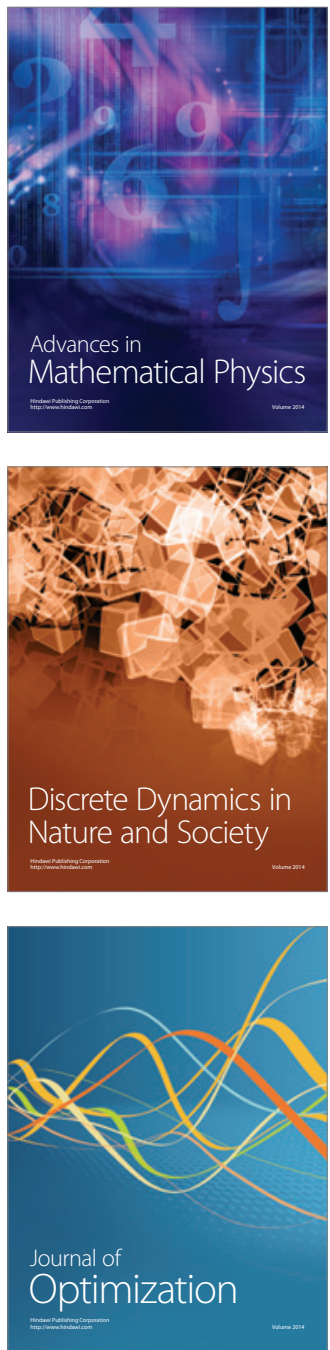